\title{
Mapa de empatía en docentes del Centro de Atención Múltiple sobre la infraestructura educativa en San Felipe, Guanajuato
}

\author{
Empathy map in teachers of the Multiple Attention Center on the educational infrastructure in \\ San Felipe, Guanajuato.
}

Alejandra Solórzano Gutiérrez ${ }^{a}$

\begin{abstract}
:
This article shows the results of an instrument used in an investigation that had the purpose of showing through an empathy map as a questioning tool, a visualization and understanding towards teachers about the physical and emotional situation in which they work within the field of special education, area in charge of the attention of students with different capacities, dimensioned within a rural community in the state of Guanajuato, Mexico. Teachers will offer an occupational perspective of their professional work within the classroom, the experiences they assimilate from it, and how the educational infrastructure influences their work.
\end{abstract}

Keywords:

Teachers; perception; empathy; education.

\section{Resumen:}

Este artículo muestra resultados de un instrumento utilizado en una investigación que tuvo la finalidad de mostrar a través de un mapa de empatía como herramienta de cuestionamiento, una visualización y comprensión hacia los docentes sobre la situación física y emotiva en la que laboran dentro en el ámbito de la educación especial, área encargada de la atención del alumnado con capacidades diferentes, dimensionado dentro de una comunidad rural en el estado de Guanajuato, México. Los docentes ofrecerán una perspectiva ocupacional de su quehacer profesional dentro del salón de clases, las experiencias que asimilan de este y cómo la infraestructura educativa influencia su labor.

Palabras Clave:

Docentes; percepción; empatía; educación

\section{Introducción}

Uno de los pilares principales para el desarrollo de una nación es la educación, el sistema educativo ofrece al alumnado de diferentes edades, condiciones y regiones dentro de la República Mexicana un servicio de calidad, en mejora continua a través de docentes preparados, materiales e infraestructura física para el completo desarrollo y fortalecimiento de sus capacidades y competencias intelectuales; destacando el acceso y la no discriminación.

Dentro de los participantes primordiales en esta ecuación educativa, se encuentra el alumnado con capacidades diferentes como usuarios, y como prestadores del servicio los docentes, ya que son la primer línea de acercamiento a ellos, aunque no tengan una autoridad sobre decisiones en muchos sentidos. Estos profesionales de la educación son quienes mostrarían una perspectiva de las situaciones que acontecen en el interior del aula de educación especial, desde lo que piensan, ven y oyen de la infraestructura, así como el ambiente en el que se desempeñan.

Comencemos por definir qué es la educación especial, cuál es el espacio destinado para este servicio, qué objetivos se plantean para los docentes de esta modalidad educativa, el sistema perceptivo y cuáles son los factores externos que influencian su quehacer. Mostrar esta información incitará en el lector, el primer paso sobre el reconocimiento hacia la empatía. 


\section{Educación Especial}

Hablar de la educación especial es referirse a aquella atención especializada y de calidad sobre los alumnos con capacidades diferentes, cuya responsabilidad monumental se encuentra orquestado por el Sistema Educativo Nacional, además de ser el encargado de darle seguimiento a la Ley General de Educación (LGE) en vigor, que establece en su capítulo VIII a la educación especial como una educación inclusiva y se refiere a ella como el "conjunto de acciones orientadas a identificar, prevenir y reducir las barreras que limitan el acceso, permanencia, participación y aprendizaje de todos los educandos, al eliminar prácticas de discriminación, exclusión y segregación" (SEP, 2019). Por lo tanto, las autoridades correspondientes buscarán asegurar la educación inclusiva en todos los tipos y niveles educativos, con el fin de favorecer el aprendizaje de todos los estudiantes, haciendo énfasis en los que están excluidos, marginados o en riesgo de estarlo.

La atención de los alumnos que presentan necesidades educativas especiales, está integrada por aquel alumnado con dificultades en el área motriz, intelectual, sensitiva, etc., integrando parte de lo que es la educación espacial; a lo largo de su historia, han brindado respuestas educativas a esta población, a través de acciones congruentes con los distintos momentos educativos; sin embargo, en los últimos años y a partir de la promoción de la integración educativa, la educación básica ha asumido esta responsabilidad (SEP, 2006).

Históricamente hablando, la educación especial institucionalizada se inició en México con la fundación de la Escuela Nacional de Ciegos, en 1870, seguida por la fundación de la Escuela Nacional de Sordos, en 1861 (SEP, 2004).

\section{Infraestructura Educativa}

La LGE establece, que las escuelas constituyen un espacio fundamental para el proceso de enseñanza aprendizaje, donde se presta el servicio público de educación por parte del Estado o por los particulares con autorización o reconocimiento de validez oficial de estudios.

Muebles e inmuebles deberán cumplir con los requisitos de calidad, seguridad, funcionalidad, oportunidad, equidad, sustentabilidad, resiliencia, pertinencia, integralidad, accesibilidad, inclusividad e higiene, incorporando los beneficios del desarrollo de la ciencia y la innovación tecnológica, para proporcionar educación de excelencia, con equidad e inclusión (SEP, 2019).

Tratándose de la educación especial, el sistema educativo en busca de una mejora continua y con la reorientación y reorganización de sus servicios, tienen como propósitos principales el combatir la discriminación, la segregación y el etiquetaje derivado de la atención a las niñas y los niños con capacidades diferentes, que se encontraban separados del resto de la población infantil y de la educación básica general; así como la atención especializada que era principalmente de carácter clínicoterapéutico y en ocasiones, atendía con deficiencia otras áreas del desarrollo como el aprendizaje de la lectura, la escritura y las matemáticas (SEP, 2006), donde la nueva organización de los servicios educativos especiales trabaja bajo la siguiente estructura:

I. Los servicios indispensables de educación especial se trasformaron en Centros de Atención Múltiple (CAM). El CAM ofrecería atención en los distintos niveles de educación básica utilizando, con las adaptaciones pertinentes, los planes y programas de estudio generales y formación para el trabajo. Asimismo, se organizaron grupos/grados en función de la edad de la población, lo cual congregó alumnos con distintas discapacidades en un mismo centro y/o grupo.

II. Los servicios complementarios se transformaron en Unidades de Servicios de Apoyo a la Educación Regular (USAER), con el propósito de promover la integración de las niñas y los niños con necesidades educativas especiales a las aulas y escuelas de educación inicial y básica regular.

III. Los Centros de Orientación, Evaluación y Canalización (COEC) y los Centros de Orientación para la Integración Educativa (COIE), se transformaron en Unidades de Orientación al Público (UOP), destinadas a brindar información y orientación a las familias y a los maestros sobre el proceso de integración educativa.

IV. Se promovió la transformación de los Centros de Atención Psicopedagógica de Educación Preescolar (CAPEP) en servicios de apoyo a la integración educativa en los jardines de niños.

Centralizando la infraestructura especial y haciendo un énfasis en este, los Centros de Atención Múltiple son un servicio escolarizado de la Dirección de Educación Especial donde se ofrece educación inicial y básica (preescolar, primaria y secundaria) de calidad a niñas, niños y jóvenes con discapacidad, discapacidad múltiple o trastornos graves del desarrollo, condiciones que dificultan su ingreso en escuelas regulares (INIFED CAM, 2013). Esta modalidad u organización que integra el sistema educativo y el organigrama de educación especial, cuenta con presencia en todos los estados de la República Mexicana, incluyendo una comunidad como lo es San Felipe, Guanajuato. 


\section{Retos del Docente}

No existe una fórmula para afrontar las diferentes situaciones a las que son sometidos los docentes, cada institución educativa es diferente, ya que posee sus ventajas y desventajas en cuanto a recursos pedagógicos, apoyo institucional o de los tutores o padres de familia, el espacio donde desarrollan sus actividades o hasta el mismo núcleo laboral puede ser visto como un foco de atención. De los docentes, se puede esperar con seguridad el desarrollo y cumplimiento de sus actividades dentro del salón de clases, ya que es una responsabilidad irrefutable con la que deben de efectuar, concretar los planes de estudio aun enfrentando las circunstancias positivas o negativas.

Dentro de la Ley General de Educación (SEP, 2019) se menciona que las maestras y los maestros son agentes fundamentales del proceso educativo $y$, por tanto, se reconoce su contribución a la transformación social y como parte de los objetivos de una revalorización de las maestras y maestros se tiene que promover lo siguiente:

- Priorizar su labor para el logro de metas y objetivos centrados en el aprendizaje de los educandos;

- Fortalecer su desarrollo y superación profesional mediante la formación, capacitación y actualización;

- Fomentar el respeto a la labor docente y a su persona por parte de las autoridades educativas, de los educandos, madres y padres de familia o tutores y sociedad en general; así como fortalecer su liderazgo en la comunidad;

- Reconocer su experiencia, así como su vinculación y compromiso con la comunidad y el entorno donde labora, para proponer soluciones de acuerdo con su contexto educativo;

- Priorizar su labor pedagógica y el máximo logro de aprendizaje de los educandos sobre la carga administrativa;

- Promover su formación, capacitación y actualización de acuerdo con su evaluación diagnóstica y en el ámbito donde desarrolla su labor;

- Impulsar su capacidad para la toma de decisiones cotidianas respecto a la planeación educativa;

Lo anterior, son algunas de las formas de apoyar la labor docente como autoridad, pero como participes de una disciplina diferente a la pedagogía, es relevante poder hacer una aportación desde nuestra área de conocimiento; y es donde la Arquitectura, la psicología ambiental deben fomentar un interés por el cambio de situaciones desfavorables, desde hacer visibles elementos tangibles como escuelas $u$ alguna otra construcción o espacio, hasta promover en un nivel antropológico características de los usuarios intangibles como estados de ánimo o percepciones.

\section{Percepción}

El termino de percepción es la característica con la cual estamos íntimamente relacionados los seres humanos, ya que somos participes de percibir a diario estímulos nuevos o cotidianos de los cuales aprendemos o ignoramos.

El proceso perceptivo es variado y complejo donde el tiempo, el contexto, el mecanismo de transmisión, la capacidad sensitiva y la interpretación de estímulos son universos diferentes, pero no trabajan aisladamente. Un determinante importante dentro del proceso perceptivo es la experiencia; cuando un estímulo es nuevo adquiere relevancia en nuestro organismo formando parte de nuestra memoria y permanece latente, cuando un estimo es reconocible por intervenciones previas el organismo compara las percepciones anteriores con las actuales para denotar cambios y marcar un referente en nuestra consciencia como un olor peculiar o un sonido distintivo.

Autores reconocen al proceso perceptivo como una sustracción del inconsciente "como un proceso construido involuntariamente en el que interviene la selección de preferencias, prioridades, diferencias cualitativas y cuantitativas del individuo acerca de lo que percibe" (Abbagnano, 1986). También se refieren a la percepción como "parte esencial de la conciencia, es la parte que consta de hechos intratables y, por tanto, constituye la realidad como es experimentada" (Carterette \& Friedman, 1982). De igual forma es aquella que "atribuye características cualitativas a los objetos 0 circunstancias del entorno mediante referentes que se elaboran desde sistemas culturales e ideológicos específicos construidos y reconstruidos por el grupo social, lo cual permite generar evidencias sobre la realidad" (Melgarejo, 1995). La percepción no es un añadido de eventos a experiencias pasadas sino una constante construcción de significados en el espacio y en el tiempo. (Merleau-Ponty, 1975) Así los diferentes autores expresan una perspectiva propia de la dimensión de la percepción. Grandjean afirma que "la percepción no es una copia auténtica del mundo exterior; el mundo externo es experimentado subjetivamente, es percibido por un proceso sensorial y modulado por un proceso puramente subjetivo". Para el autor, "la percepción proporciona como regla general, la información necesaria para una reacción adecuada al mundo exterior" (Grandjean, 1998)

Dentro del universo perceptivo podemos denotar diferencias, en su mayoría culturalmente hablando; la 
percepción está matizada y restringida por las demarcaciones sociales que determinan rangos de sensaciones, sobre el margen de posibilidades físicocorporales; así la habilidad perceptual real queda subjetivamente orientada hacia lo que socialmente está "permitido" percibir.

La medición de la percepción en algunas investigaciones puede tratarse desde análisis factoriales (Ocampo \& Palos, 2007)si se quiere cuantificar tratándose de "un modelo estadístico que representa las relaciones entre un conjunto de variables, plantea que estas relaciones pueden explicarse a partir de una serie de variables no observables (latentes) denominadas factores, siendo el número de factores substancialmente menor" (Ferrando \& Anguiano-Carrasco, 2010); y que es comúnmente usado en la psicología. Investigaciones donde la evaluación o valoración se da por medio de una selección de ítems dentro de una escala previamente formulada por ejemplo la percepción del estrés (Cabanach et al., 2010), o la medición más común de la percepción que es por medio de la observación y cuestionamiento de participantes con diferentes métodos de acercamiento, ya que es un proceso perceptivo e interpretativo es individual.

\section{Contexto Social}

Existen una cantidad innumerable de influencias a las cuales se exponen a los docentes, dentro del Modelo de Atención de los Servicios de Educación Especial o MASEE (SEP, 2011) se plantean prioridades en cuanto a las relaciones que se fomentan el en ámbito educativo en la modalidad especial, si bien se habla del Centro de Atención Múltiple como una institución responsable de cumplir con estas aseveraciones, también es justo reconocer la labor en conjunto de quienes participan al interior de ese núcleo de servicio, que llevan parte de la responsabilidad para que toda ejecución o planteamiento se cumpla satisfactoriamente con su propósito en medida de lo posible. Es importante rescatar y visibilizar estos objetivos o prioridades ya que abren un panorama de áreas de oportunidad a trabajar, apoyar o reforzar dentro de las relaciones interpersonales en caso de que no se estén resolviendo adecuadamente, siendo el primer paso de la empatía reconocer cuales son los lazos que se manejan dentro de un campo educativo especial.

En relación con las alumnas y los alumnos:

- Promover la inclusión educativa y/o laboral.

- Ofertar una atención integral en educación inicial, básica y formación para la vida y el trabajo y por una propuesta didáctica que privilegia el aprendizaje y el desarrollo de competencias desde los campos de formación.
- Brindar espacios educativos para el desarrollo pleno del alumnado en temas como la sexualidad y el desarrollo de habilidades artísticas, culturales, deportivas y recreativas, favoreciendo en ellos una educación integral.

- Desarrollar competencias laborales en jóvenes con discapacidad para la realización de actividades productivas las cuales les permitan lograr su independencia y autonomía de acuerdo con sus condiciones de vida particular y contextual.

En relación con las familias de las alumnas y los alumnos:

- Fortalecer la participación de las familias en el proceso educativo de sus hijos.

- Trabajar de manera vinculada con las familias: elaborar estrategias de "apoyo en casa" para fortalecer la independencia y la inclusión social y laboral.

- Desplegar acciones para promover la mejora de la calidad de vida familiar.

- Reconocer la participación de las familias como un derecho.

- Valorar las culturas de las familias.

- Ofrecer apoyo emocional e informar a las familias sobre temas relacionados con la discapacidad, el desarrollo humano y la sexualidad, entre otros.

- Proporcionar asesoría y orientación sobre las diferentes alternativas de formación para el trabajo y de inclusión educativa y laboral.

- Establecer compromisos de corresponsabilidad entre el sector educativo, el productivo, la familia y el alumnado.

\section{En relación con los docentes:}

- Impulsar una cultura del trabajo en equipo para movilizar a la comunidad educativa hacia la mejora y reestructuración de las prácticas, las políticas y cultura del servicio.

- Implantar los compontes de la gestión educativa estratégica, como condición necesaria para transformar la gestión escolar y pedagógica.

- Proporcionar orientación y asesoría a profesionales que apoyan la inclusión educativa de alumnos y alumnas con discapacidad sobre diferentes recursos, estrategias y metodologías de atención.

\section{Con la sociedad:}

- Coadyuvar en los procesos de inclusión social para favorecer la participación de los alumnos y alumnas en la comunidad. 
- Asumir el proceso de atención educativa, como una tarea social imprescindible para proyectar una sociedad respetuosa de la diversidad y en contra de cualquier acto de discriminación y exclusión.

\section{Contexto Ambiental}

El contexto como panorama exterior tiene un valor importante cuando ubicamos el elemento a estudiar en ambientes reales ya que se tiene un visualización y análisis más completo, complejo y significativo, que si se estudiaran los elementos aislados. La interacción con el ambiente proporciona al individuo una gran variedad de señales sensoriales o retroalimentación (por ejemplo, visuales, auditivas y táctiles) acerca de la naturaleza del ambiente. Donald Berlyne propuso cuatro variables para dimensionar la estimulación ambiental: novedad, complejidad, sorpresa e incongruencia. (Berlyne, 1960) Joachim descubrió que la manera en que un individuo explora el ambiente depende de la novedad de sus características (Wohlwill, 1996). Para la medición o valoración de la percepción ambiental, nos encontramos con que no se puede lograr el control experimental sobre la estimulación ambiental como se obtendrían en un laboratorio, por ejemplo, las simulaciones corren el riesgo de restar validez. "La escala multidimensional describe las interrelaciones de las complejas fuentes de datos, que permite identificar aquellas características ambientales (por ejemplo, diversidad, calor, tamaño, complejidad y familiaridad) que predominan en la percepción de muchas personas (Green \& Rao, 1972)

Una de las principales funciones psicológicas de la percepción ambiental es dirigir y regular las actividades que constituyen la vida diaria del individuo (Ittelson, Franck, \& O'Hanlon, 1976) la percepción del ambiente proporciona las bases para conocer el mundo que habitamos y ayuda al individuo a orientarse y dirigir sus actividades cotidianas.

Con lo anterior y regresando al punto inicial San Felipe una población del estado mexicano de Guanajuato; localizada al noroeste y con una población total en viviendas particulares habitadas de 113,109 en el año 2015 (INEGI, 2015) es una comunidad como muchas dentro del estado y de la República Mexicana, que, así como tiene sus buenas prácticas, también se encuentra desfavorecido o con desventajas sobre otras poblaciones. Actualmente cuenta con solo un CAM registrado en el portal del Sistema de Información y Gestión Educativa del Gobierno de México; bajo un sostenimiento público, con turno matutino el CAM Gabriela Brimmer. (SIGED, 2020)

Las estadísticas del CAM mencionado anteriormente y bajo los requisitos de los "Cuestionarios del formato 911" con fuente del Ciclo inicio 19-20 se presentan:
- 19 niñas

- 42 niños

- 1 maestro

- 5 maestras

- 13 grupos

- 6 aulas

- 1 cancha

- 16 computadoras

- 1 patio

- 0 docentes especiales de educación física

- 0 docentes especiales de educación artística

- 0 docentes especiales de educación en tecnologías

- 0 docentes especiales de idiomas.

Dentro de los datos geográficos que deben ser mencionados para comprender el contexto se encuentra el clima anualmente ya que juega un papel importante dentro de la habitabilidad de un salón de clases; se presentan a continuación un resumen del clima en San Felipe, Guanajuato donde podemos visualizar máximas y mínimas anuales de la nubosidad, precipitación, humedad, temperatura hasta valoración del turismo fomentando su llegada; de igual forma se recomienda realizar una visita al sitio web meteorológico para reconocer algún otro dato especifico, histórico o comparativa con respecto a la comunidad.

\section{Figura 1.}

Resumen de datos climáticos San Felipe, Guanajuato.

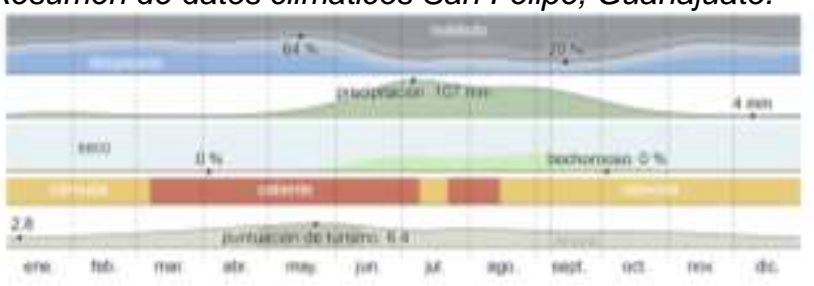

Fuente: (Weather Spark, 2020)

\section{Mapa de Empatía}

Dentro la disciplina de la Mercadotecnia, existen estrategias factibles de aplicar en áreas completamente similares y distintas a la vez; el Mapa de Empatía a grandes rasgos es un recurso que sirve para diseñar el perfil de tu usuario con base en sus sentimientos; el termino de empatía según el diccionario de la lengua española el termino de empatía se refiere a la "capacidad de identificarse con alguien y compartir sus sentimientos". (RAE, 2020) Ese sentimiento que describe la habilidad de comprender el estado emocional del otro al colocarse en el lugar de él permite ver las situaciones sobre perspectivas diferentes y entender las razones por las cuales los individuos actúan de embates innecesarios. Ahora, imaginen retirar esos sentimientos del plano de las 
ideas y colocarlos sobre el papel, dividiéndolos en categorías como dolores, necesidades y sentimientos, en cuadrantes que facilitan la visualización.

El Mapa de Empatía fue diseñado por Dave Gray, autor y fundador de XPLANE, una firma consultora que utiliza herramientas visuales para potenciar la visión estratégica de sus clientes. La primera versión de este Mapa de Empatía fue presentada por Gray en su libro Game Storming, que se ha convertido en una enciclopedia práctica de herramientas visuales y ejercicios creativos para resolver problemas en diferentes etapas del proceso de Design Thinking.

\section{Figura 2.}

Mapa de Empatía Canvas.

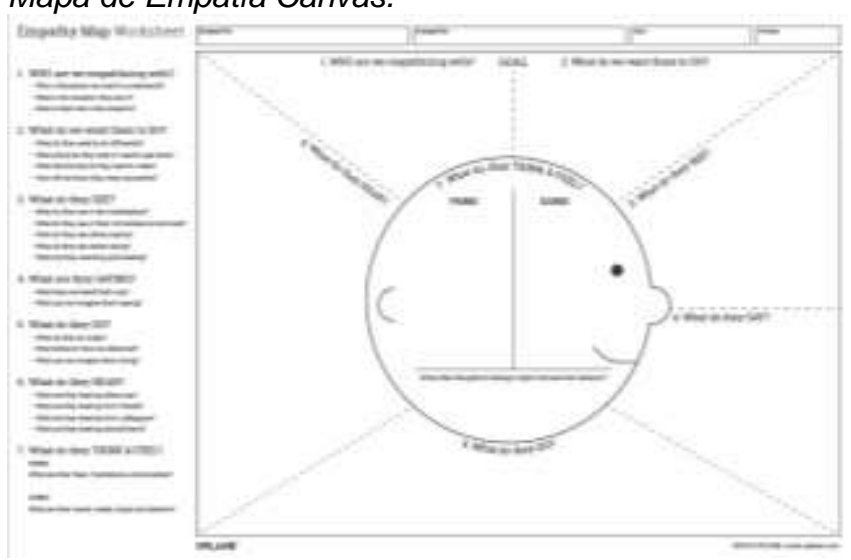

Fuente: (XPLANE, 2019).

Dentro del CANVAS o platilla desarrollada para el Mapa de empatía podemos localizar 6 elementos relevantes y ubicados en disposiciones específicas y estratégicas, con la intención de acercarnos a los usuarios que en este caso particular se refiere a los maestros de una forma racionalizada. A continuación, se muestra aquellos acercamientos sobre cada sección del mapa de empatía y una forma de poder abordarlo por medio de otras interrogantes. (RD Station, 2017).

1. ¿Qué ve? Ese primer cuadrante se refiere a los estímulos visuales que tu persona recibe. Intenta responder a preguntas como:

- ¿Cómo es el mundo en que la persona vive?

- ¿Cómo son sus amigos?

- ¿Qué es lo más común en su cotidiano?

2. ¿Qué escucha? Aquí, piensa en lo que tu persona oye no solamente en sentido sonoro, de música o conversaciones, sino también en las influencias de diversas fuentes, como medios de comunicación. Puedes buscar responder a preguntas como:

- ¿Cuáles personas e ideas la influencian?

- ¿Cuáles productos de comunicación consume?
3. ¿Qué piensa y siente? Son las ideas que despierta en la mente de los usuarios.

- ¿Cómo la persona se siente en relación con el mundo?

- ¿Cuáles son sus preocupaciones?

- ¿Cuáles son sus sueños?

4. ¿Qué habla y hace? Este punto se relaciona al uso del servicio, desde cuando la persona toma la decisión de pertenecer. Para entender lo que tu persona habla y hace, presta atención a su comportamiento: el discurso que hace y lo que practica.

- ¿Sobre qué acostumbra a hablar?

- Al mismo tiempo, ¿Cómo actúa?

- ¿Cuáles son sus hobbies?

5. ¿Cuáles son sus dolores? Corresponde a las dudas y obstáculos que el usuario debe superar

- ¿De qué tiene miedo?

- ¿Cuáles son sus frustraciones?

- ¿Qué obstáculos debe traspasar para conseguir lo que desea?

6. ¿Cuáles son sus necesidades? Tiene relación con lo que puedes colocar en práctica para sorprender a tu usuario, mostrándole posibilidades. Cuestiona sobre:

- ¿Qué es éxito para tu persona?

- ¿A dónde este quiere llegar?

- ¿Qué acabaría con sus problemas?

\section{Método}

Para el Mapa de Empatía desarrollado y adaptado para los docentes del CAM en San Felipe, Guanajuato, se realiza bajo el siguiente cuestionario en el que se incluyen los principios básicos como ¿Qué ve? ¿Qué escucha? ¿Qué piensa y siente? ¿Qué habla y hace? ¿Cuáles son sus necesidades? Incluyendo una revisión de la calidad en sus relaciones interpersonales con los demás participantes, así como el objetivo primordial que es conocer sobre la infraestructura para comprender la situación de las instalaciones y habitabilidad de ese espacio. A continuación, se presenta el modelo de preguntas:

1. ¿Qué opinas sobre el aspecto actual del salón de clases?

2. ¿Cuál fue la última mejora realizada en el salón de clases?

3. ¿Consideras que el salón de clases necesita alguna mejora? ¿Cuál sería? 
4. ¿Qué sensación anímica te transmite el interior del salón?

5. ¿Cuál es la temperatura que predomina al interior del salón?

6. ¿Existe suficiente iluminación natural y artificial para realizar sus actividades? ¿Qué sugieres para mejorar?

7. ¿Existe suficiente espacio al interior del salón para realizar sus actividades? ¿Qué sugieres para mejorar?

8. ¿Existe suficiente mobiliario para realizar sus actividades? ¿Qué sugieres para mejorar?

9. ¿Existen suficientes entradas de aire hacia el interior del salón? ¿Qué sugieres para mejorar?

10. ¿Tus alumnos se distraen mucho en clases? ¿A qué se debe?

11. Para impartir tus clases ¿Requieres incrementar el volumen de tu voz?

12. ¿Existe algún sonido exterior o interior que compita con tu voz cuando impartes clases? ¿Cuál es?

13. Podrías breve mente describir desde tu posición como maestro(a) ¿Cuáles son tus actividades cotidianas al interior del salón en un día de clases?

14. ¿Cuáles son las actitudes de tus alumnos durante las clases?

15. ¿Cuál es la mayor dificultad que encuentras para realizar tus actividades al interior?

16. ¿Han tomado en cuenta tus superiores las sugerencias de los docentes? ¿A qué se debe?

17. En la escala del 0 al 5 cuan satisfactoria/pésima es tu relación con los alumnos.

18. En la escala del 0 al 5 cuan satisfactoria/pésima es tu relación con los tutores o padres de familia.

19. En la escala del 0 al 5 cuan satisfactoria/pésima es tu relación con los demás docentes.

20. En la escala del 0 al 5 cuan satisfactoria/pésima es tu relación con los directivos escolares.

\section{Resultados y Discusión}

Generar empatía es el primer paso del reconocimiento de las condiciones de las cuales no somos participes, pero podemos adquirir conciencia de las necesidades, retos, desventajas y aciertos dentro de este tipo de infraestructura educativa, que añadiendo un valor significativo como caso d estudio.

Como parte de un resumen sobre las respuestas de los participantes mostramos aquella más destacadas que ofrece una retroalimentación.

1. Es muy reducido, con iluminación y accesibilidad deficiente para todos los alumnos; deteriorado, bajos recursos materiales.
2. Cambio de piso de concreto a vitro piso; Cambio de mobiliario y de focos.

3. Ampliación de ventanas para permitir más iluminación natural y la implementación de una rampa ya que los escalones son muy altos para los alumnos con sillas de ruedas o muletas; elementos de protección, reducción de ventanas, iluminación en el acceso, cambio de mobiliario.

4. Desmotivación; abandono; soledad; frustración y desesperanza.

5. Frio; El clima que predomine en el exterior; El municipio suele ser extremo por lo que sí es tiempo de frio hace mucho frio y en tiempo de calor hace mucho calor.

6. No, las instalaciones eléctricas son malas por lo que no hay iluminación artificial y la luz natural no es suficiente; Corresponde dar un mantenimiento continuo y constante; Reubicar ciertas ventanas.

7. No, el salón está divido para que otro docente trabaje también por lo que el espacio es muy reducido y facilita la atención dispersa de los alumnos; Los espacios son reducidos con el mobiliario y los armarios de materiales.

8. No, el salón no cuenta con libreros por lo que el material está revuelto y no es de fácil acceso para todos los alumnos y las mesas no están adecuadas para todo el grupo; Reordenarlos y priorizar; brindar suficientes sillas y mesas para la cantidad de alumnos que se atienden.

9. Sí, reducir el tamaño de los paneles de las ventanas ya que o se vuelve muy frío o caluroso; Por seguridad no se abren las ventanas constantemente, nos apoyamos de ventiladores.

10.Sí, el espacio no facilita el desarrollo de las actividades, debido al ruido del otro docente en el salón; El espacio es muy reducido y se acumulan las voces o no se escuchan completamente.

$11.100 \%$ de los docentes incrementan el volumen de su voz para impartir su clase.

12. Sí, la clase y voz del otro maestro; Exterior ninguno.

13. La atención individual a alumnos con algún tipo de discapacidad, trabajo en subgrupos de hasta 7 niños con problemas de aprendizaje y atención a madres de familia y docentes de primaria para sugerencias; Reviso el estado del salón, selecciono mis materiales, imparto las clases, atiendo a los niños, apoyo en sus actividades y corroboró sus resultados

14. Mayormente positivas ante el trabajo; Colaboración; Aceptación.

15. Falta de espacio; Orden; Seguridad;

16. No, comentan que las condiciones y solución de ellas, no depende de las supervisiones escolares, si no de los recursos de programas federales para la 
educación; Las delegaciones dan respuestas ambiguas a las peticiones.

17. $100 \%$ de los docentes consideran satisfactoria su relación con los alumnos.

$18.83 .33 \%$ considera satisfactoria y un $16.66 \%$ considera buena su relación con los tutores o padres de familia.

19.66.66\% considera satisfactoria y un $33.33 \%$ considera buena su relación con los demás docentes o compañeros de trabajo.

$20.83 .33 \%$ considera buena y un $16.66 \%$ considera regular su relación con los directivos escolares

Discutir sobre los usos de otras herramienta pertenecientes a diferentes disciplinas como en el caso particular de esta investigación, que se trata de una estrategia o metodología del marketing puede generar diferentes opiniones, la validación de esta siempre representa un reto más cuando esta es novedosa o poco explorada en tu área de conocimiento, cuando los resultados a obtener expresan los objetivos planteados es difícil negarse a probar con ellas. El mapa de empatía cumplía con la función de visibilizar las situaciones de los docentes desde su particular punto de vista o perspectiva donde no se requiere forzosamente datos cuantitativos ya que no lo ameritan por ahora, es importante destacar que este podría ser un primer paso para vigilar aquellas flaquezas que podrían detectarse y poder actuar en ellas de ser correctas y dar comienzo a otro tipo de evaluaciones o investigaciones sobre este campo de la educación especial, de la infraestructura educativa.

\section{Referencias}

Abbagnano, N. (1986). Diccionario de filosofía (Segunda ed.). México: Fondo de Cultura Económica.

Berlyne, D. E. (1960). Conflict arousal and curiosity. Nueva York: McGraw-Hill.

Cabanach, R. G., Valle, A., Rodríguez, S., Piñeiro, I., \& Freire, C. (2010). Escala de afrontamiento del estrés académico (A-CEA). Revista Iberoamericana de Psicología y Salud, 51-64.

Carterette, E., \& Friedman, M. (1982). Manual de percepción : raíces históricas y filosóficas. México: Trillas.

Ferrando, P. J., \& Anguiano-Carrasco, C. (2010). El análisis factorial como técnica de investigación psicología. Papeles del psicólogo, 18-33.

Grandjean, E. (1998). Manual de ergonomia: adaptando o trabalho ao homem. Porto Alegre: Artes Médicas.

Green, P. E., \& Rao, V. R. (1972). Applied Multidimensional Scaling: A comparison of approaches and Algorithms. Nueva York: Rinehart y Winston.

INEGI. (2015). Población. Banco de Indicadores. Ciudad de México, México.

INIFED CAM. (15 de 10 de 2013). Criterios Normativos INIFED. Obtenido de Instituto Nacional de la Infraestructura Física Educativa: Normativa Técnica: https://www.gob.mx/cms/uploads/attachment/file/106549/XVIII_D_-
_Criterios_de_Dise_o_Arquitect_nico_para_Centros_de_Atenci_n_M ltiple.compressed.pdf

Ittelson, W. H., Franck, K. A., \& O'Hanlon, T. J. (1976). The Nature of Environmental Experience. New York: Plenum.

Melgarejo, L. M. (1995). Los colores lacandones: un estudio sobre percepción visual. México.

Merleau-Ponty, M. (1975). Fenomenología de la percepción. Barcelona: Península.

Ocampo, D. B., \& Palos, P. A. (2007). Escala de percepción del control parental de niños. Investigación Universitaria Multidisiplinaria , 26-34.

RAE. (2020). Empatía. Obtenido de Real Academia Española: https://dle.rae.es/empat\%C3\%ADa

RD Station. (7 de Diciembre de 2017). Mapa de empatía: qué es y 6 pasos para creas uno de calidad. Obtenido de Blog de Marketing Digital de Resultados: https://www.rdstation.com/mx/blog/mapa-deempatia/\#: :text=E1\%20mapa\%20de\%20empat\%C3\%ADa\%20es\%20 un\%20formato $\% 20$ que $\% 20$ busca $\% 20$ describir,y\%20como\%20relacion arse $\% 20$ con $\% 20 \% \mathrm{C} 3 \% \mathrm{~A} 91$.

SEP. (2004). Licenciatura en Educacion Especial. Plan de Estudios. Mexico. Recuperado el 2020, de Plan de Estudios.

SEP. (2006). Orientaciones generales para el funcionamiento de los servicios de educación especial. Recuperado el 2020, de Secretaría de Educación Pública: https://www.educacionespecial.sep.gob.mx/pdf/publicaciones/libromor ado.pdf

SEP. (Septiembre de 2011). MASEE. Modelo de Atención de los Servicios de Educación Especial. Ciudad de México, México.

SEP. (30 de Septiembre de 2019). Ley General de Educación. Ley General de Educación. México: Diario Oficial de la Federación.

SIGED. (2020). Sistema de Información y Gestión Educativa. Obtenido de Consulta de escuelas: https://www.siged.sep.gob.mx/SIGED/escuelas.html

Weather Spark. (2020). Promedio: San Felipe, Etsado de Guanajuato, México. Obtenido de Weather Spark: https://es.weatherspark.com/y/4629/Clima-promedio-en-San-FelipeM\%C3\%A9xico-durante-todo-el-a\%C3\%B1o

Wohlwill, J. F. (1996). The physical environment: A problem for a psychology of simulation. Journal of social issues, 29-38.

XPLANE. (2019). Empathy Map Worksheet. Obtenido de Xplane: https://xplane.com/es/worksheets/empathy-map-worksheet/ 